\title{
Crystal structure of human BS69 Bromo-ZnF-PWWP reveals its role in $\mathrm{H} 3 \mathrm{~K} 36 \mathrm{me} 3$ nucleosome binding
}

Cell Research (2014) 24:890-893. doi:10.1038/cr.2014.38; published online 28 March 2014

\section{Dear Editor,}

The eukaryotic genome is packed into highly compacted chromatin, the basic unit of which is a nucleosome composed of 147 base pairs of DNA wrapped around the histone octamer [1]. Post-translational modifications along histone $\mathrm{N}$-terminal tails can be recognized by various 'reader' modules [2]. PWWP domain, one type of 'reader' modules, has been identified to recognize H3K36me3 in the peptide and nucleosome context [3-5].

BS69 (ZMYND11), a multidomain protein, contains a PHD finger, a bromodomain (Bromo) and a PWWP domain at the N-terminal half and a MYND domain at the C-terminus (Figure 1A). BS69 associates with chromatin and functions as a transcription co-repressor by interacting with various transcription and chromatin remodeling factors [6-9]. However, the molecular mechanisms by which BS69 engages in chromatin association and transcription repression remain elusive. Here, we determined the $1.9 \AA$ crystal structure of BS69 Bromo-ZnFPWWP (Asn154-Gly371), which revealed a previously uncharacterized $\mathrm{CCCH}$-type zinc finger $(\mathrm{ZnF})$ between Bromo and PWWP domain (Figure 1B and Supplementary information, Table S1). Structure comparison using Dali sever [10] shows that $\mathrm{ZnF}$ folds into a novel fold, in which Pro279 adopts a cis conformation, facilitating an $\alpha$-turn formation between the third and fourth zinc-binding ligands (Figure 1C and Supplementary information, Figure S1). ZnF unifies the Bromo and PWWP modules to form an integrated supramodule via extensive polar and hydrophobic intermodule interactions (Figure 1D and Supplementary information, Figure S2). The unified organization of Bromo-ZnF-PWWP in solution was also confirmed by small angle X-ray scattering (Supplementary information, Figure S3). Sequence alignment and SMART database [11] search reveals that the combination of Bromo-ZnF-PWWP is evolutionarily conserved and only exists in BS69 and ZMYND8 (Supplementary information, Figure S4). Although we failed to detect the binding substrate of Bromo (see below), the supramodule probably functions as an integrated unit to associate with chromatin, which maybe involves the preceding PHD finger.

The PWWP domain is composed of a five-stranded antiparallel $\beta$-barrel followed by a helical region. A conserved and hydrophobic aromatic cage locates at one end of the $\beta$-barrel (Figure 1E and Supplementary information, Figure S5A), which is a common molecular architecture for recognition of methyl-lysine [2]. We therefore explored the binding properties of BS69 to methylated histone peptides. Bromo-ZnF-PWWP significantly bound to methylated $\mathrm{H} 3 \mathrm{~K} 36$ peptides in histone peptide arrays (Supplementary information, Figure S5C). Fluorescence polarization assays (FPAs) established that Bromo-ZnFPWWP preferred to bind H3K36me3 with binding affinity in the millimolar range. Mutation of Trp294, which is located in the aromatic cage, abolished this interaction (Figure 1F). In addition, we found that full-length BS69 specifically interacted with $\mathrm{H} 3 \mathrm{~K} 36 \mathrm{me} 3$ in native nucleosome co-immunoprecipitation (co-IP) experiments. The binding to acetylated $\mathrm{H} 4$ or $\mathrm{H} 3 \mathrm{~K} 36 \mathrm{ac}$ peptides, which was observed in the histone peptide array, was excluded by FPAs (Figure 1F) and native nucleosome co-IP with an anti-acetyl lysine antibody (Figure 1G). Compared with Brpf1 PWWP/H3K36me3 complex [3], BS69 has an additional hydrophobic residue Met288 in the aromatic cage, but lacks the insertion motif between $\beta 2$ and $\beta 3$, which forms a shallow groove to accommodate histone peptide (Supplementary information, Figure S5A and S5B). The structural differences indicate that the PWWP domain of BS69 may bind H3K36me3 in a slightly different manner.

Electrostatic potential surface of Bromo-ZnF-PWWP shows a basic region comprising Lys287, Lys289, His336, Lys338, Arg339, Lys344 and Lys345 surrounding the aromatic cage (Figure $1 \mathrm{E}$ and Supplementary information, Figure S6A). These residues are highly conserved in evolution, indicating that this region may serve as a DNA-binding surface (Supplementary information, Figure S4). Electrophoretic mobility shift assays (EMSAs) showed that Bromo-ZnF-PWWP bound to doublestrand DNA (dsDNA) without sequence preference. Ala- 
A

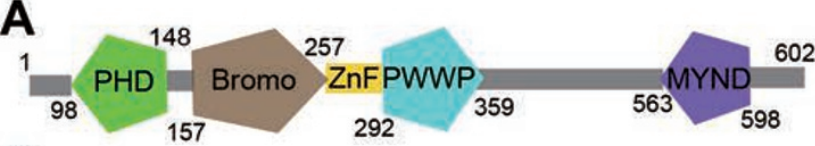

B Bromo

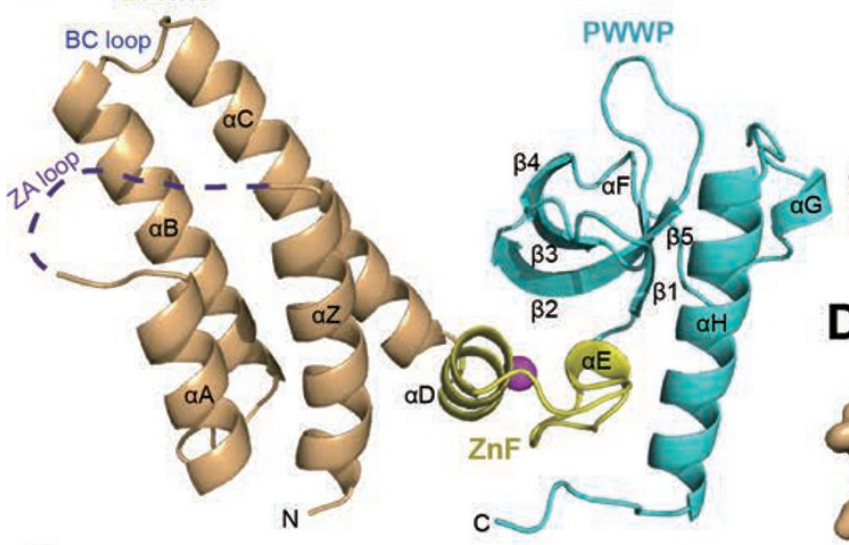

$\boldsymbol{F}_{100}$

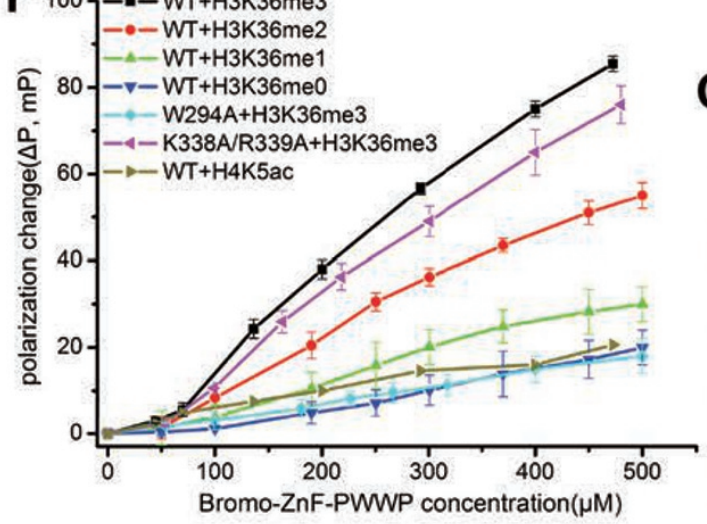

I

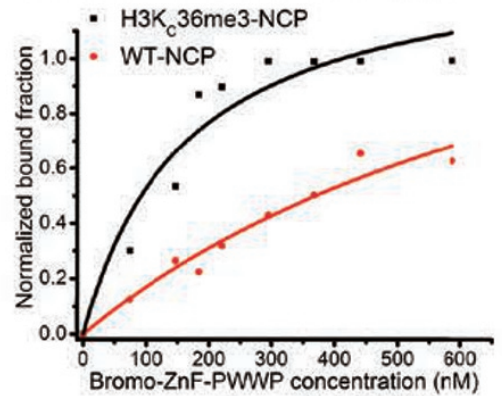

Bromo-ZnF-PWWP

H3K 36 me3-NCP

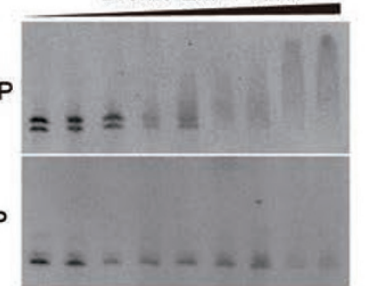

WT-NCP
C

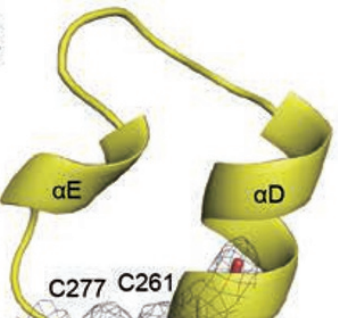

H281 C258 N/ /

D Bromo
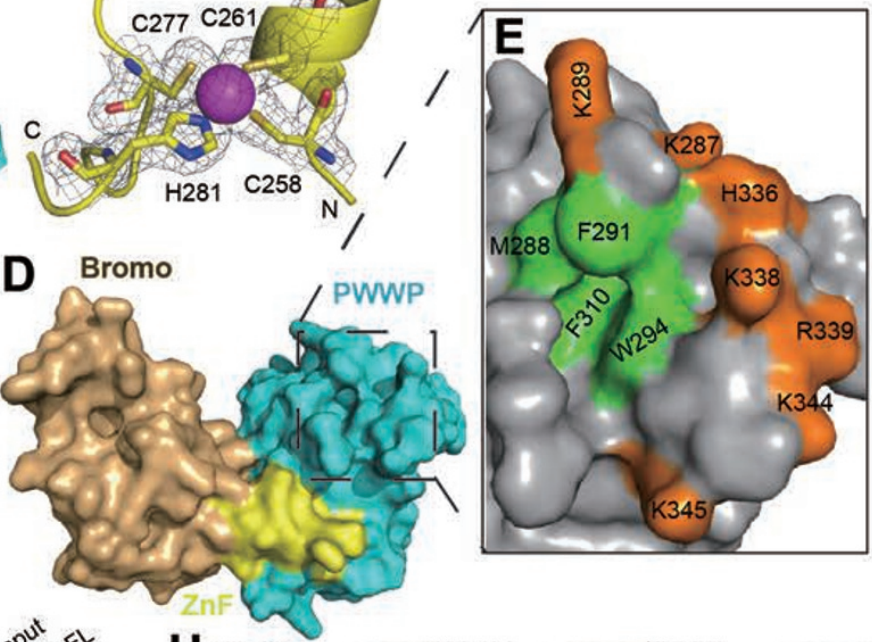

G

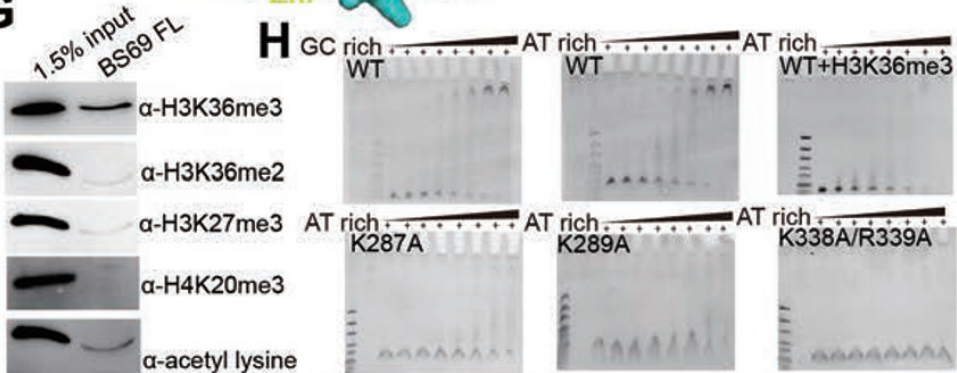

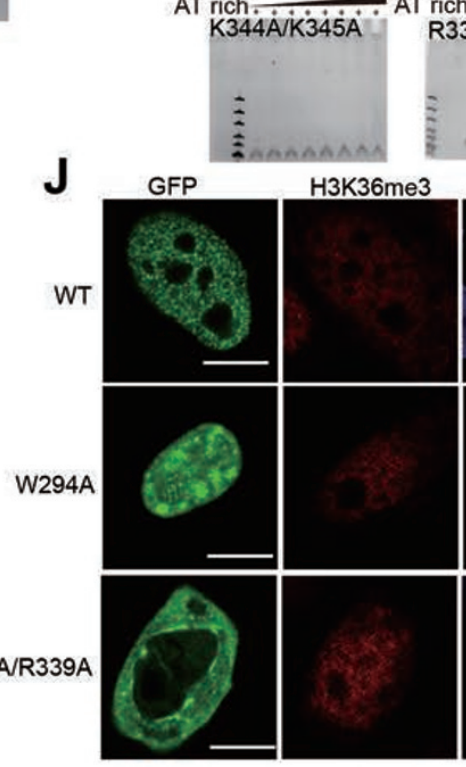

R34A $\because \because 11$

K338A/R339A
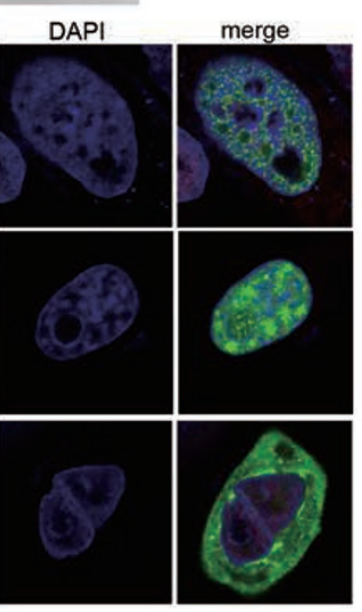
Figure 1 Structure of BS69 Bromo-ZnF-PWWP and its binding properties to H3K36me3 nucleosomes. (A) Schematic diagram showing the domain organization of BS69. (B) Ribbon representation of the overall structure of Bromo-ZnF-PWWP. (C) Residues between Bromo and PWWP domain fold into an uncharacterized zinc finger. The 2Fo-Fc omit electron density of the zinc ion and coordinating residues are contoured at 1.0б. (D, E) Surface representation of Bromo-ZnF-PWWP. The aromatic cage (green) and basic residues (orange) are shown in E. (F) FPAs of Bromo-ZnF-PWWP interacting with histone-derived peptides. (G) Native nucleosome co-IP of BS69 with nucleosomes in 293T cells. The two bands in the last panel represent acetylated H3 (upper) and H4 (lower), respectively. The signal intensity of the upper band is likely due to the interaction of H3K36me3 and BS69. (H) EMSA of Bromo-ZnF-PWWP and its mutants with dsDNA. (I) Nucleosome mobilization assays of Bromo-ZnF-PWWP with reconstituted WT- and H3K 36 me3-NCP. Band densities corresponding to free nucleosomes are analyzed using Image $\mathrm{J}$ and normalized to bound fractions. (J) Cellular localization of WT and mutant EGFP-BS69. HeLa cells ectopically expressing GFP-BS69 (green) were stained for H3K36me3 (red) and DNA (blue). Scale bar, $10 \mu \mathrm{m}$.

nine mutations of the residues in the basic region reduced the DNA-binding capacity, whereas the mutation of nonconserved Arg334 had little effect (Figure 1H). The interactions were further confirmed by FPAs with the $\mathrm{Kd}$ in the 30-60 $\mu \mathrm{M}$ range (Supplementary information, Figure S6B-S6D). These results showed that BS69 PWWP domain interacts with dsDNA nonspecifically through a conserved basic region.

The regions found to be crucial for $\mathrm{H} 3 \mathrm{~K} 36 \mathrm{me} 3$ and dsDNA binding are in close proximity. FPAs revealed that the DNA binding-deficient mutant K338A/R339A could interact with H3K36me3 peptide (Figure 1F), and the W294A mutant that was unable to bind to H3K$36 \mathrm{me} 3$ showed a dsDNA-binding affinity comparable to wild-type Bromo-ZnF-PWWP (Supplementary information, Figure S6C and S6D). EMSA confirmed that Bromo-ZnF-PWWP could still interact with dsDNA in the presence of $\mathrm{H} 3 \mathrm{~K} 36 \mathrm{me} 3$ peptide (Figure 1H). These data suggest that the histone-binding and DNA-binding regions of BS69 function in a compatible way. We therefore assume that the bivalent interactions may work cooperatively in the nucleosome context. Nucleosome mobilization assays showed that Bromo-ZnF-PWWP interacts with reconstituted nucleosome core particles (NCP) carrying a methyl-lysine analog at position 36 of H3 (H3KC36me3-NCP). The apparent Kd are $\sim 0.16 \mu \mathrm{M}$ and $0.95 \mu \mathrm{M}$ for H3KC36me3- and wild-type (WT)-NCP, respectively, strikingly higher than the binding affinity for H3K36me3 peptide and dsDNA alone (Figure 1I). Native nucleosome interaction experiments confirmed that BS69 interacted with nucleosome through DNAand H3K36me3-binding activities in vitro and in vivo (Supplementary information, Figure S5). Furthermore, cellular localization experiments also established that BS69 was recruited to H3K36me3 loci. Mutations of the residues involved in $\mathrm{H} 3 \mathrm{~K} 36 \mathrm{me} 3$ and DNA binding affect the colocalization of BS69 with H3K36me3, especially the W294A and K338A/R339A mutants, which showed diffused distribution patterns throughout the nucleoplasm and cytoplasm, respectively (Figure 1J and Supplementary information, Figure S7).
Targeted recruitments of repressors to previously active genes, which are enriched with $\mathrm{H} 3 \mathrm{~K} 36 \mathrm{me} 3$, for de novo repression are often required for lineage specification and such situation has already been observed in PHF19 [12]. We propose that BS69 specifically associates with H3K36me3-enriched chromatin through the PWWP domain, which facilitates the recruitment of MYND-bound transcription and chromatin remodeling factors including EZH2, HDAC1, Brg1 and E2F6 to target gene loci, thereby repressing target gene transcription.

\section{Accession number}

The atomic coordinates of BS69 Bromo-ZnF-PWWP are deposited in the Protein Data Bank under accession number 4NS5.

\section{Acknowledgments}

We thank Prof Jianye Zang, Dr Zhenhua Shao, Minhao Wu and the staff at BL17U of Shanghai Synchrotron Radiation Facilities for their kind help in the X-ray data collection and processing. We thank Prof Yide Mei, Ke Ruan and Jun Wan for helpful discussions. We also thank Beibei Zhang, Dr Huijuan Yu and Peng Xia for technical assistance. This work was supported by the National Basic Research Program of China (973 Program; 2011CB966302, 2012CB917201 and 2011CB911104), the National Natural Science Foundation of China (31170693, 31330018 and 31270760), the Chinese Academy of Sciences (KJZD-EW-L05), the Strategic Priority Research Program of the Chinese Academy of Sciences (XDB08010100), the Science Foundation for The Excellent Youth Scholars of Ministry of Education of China (20120001120095) and Anhui Natural Science Foundation (1208085MC38).

Juncheng Wang ${ }^{1}$, Su Qin ${ }^{1}$, Fudong $\mathrm{Li}^{1}$, Sai $\mathrm{Li}^{1}$, Wei Zhang ${ }^{2}$, Junhui Peng ${ }^{1}$, Zhiyong Zhang ${ }^{1}$, Qingguo Gong ${ }^{1}$, Jihui $\mathrm{Wu}^{1}$, Yunyu $\mathrm{Shi}^{1}$

\footnotetext{
${ }^{I}$ Hefei National Laboratory for Physical Sciences at Microscale and School of Life Sciences, University of Science and Technology of China, Hefei, Anhui 230026, China; ${ }^{2}$ Biomedical Research Institute, ShenzhenPKU-HKUST Medical Center, Shenzhen, Guangdong 518036, China

Correspondence: Jihui $\mathrm{Wu}^{\mathrm{a}}$, Yunyu $\mathrm{Shi}^{\mathrm{b}}$

aE-mail:wujihui@ustc.edu.cn

bE-mail: yyshi@ustc.edu.cn
} 


\section{References}

1 Luger K, Mader AW, Richmond RK, et al. Nature 1997; 389:251-260.

2 Taverna SD, Li H, Ruthenburg AJ, et al. Nat Struct Mol Biol 2007; 14:1025-1040.

3 Vezzoli A, Bonadies N, Allen MD, et al. Nat Struct Mol Biol 2010; 17:617-619.

4 van Nuland R, van Schaik FM, Simonis M, et al. Epigenetics Chromatin $2013 ; \mathbf{6}: 12$.

5 Eidahl JO, Crowe BL, North JA, et al. Nucleic Acids Res 2013; 41:3924-3936.
6 Ladendorff NE, Wu S, Lipsick JS. Oncogene 2001; 20:125-132.

7 Masselink H, Vastenhouw N, Bernards R. Cancer Lett 2001; 171:87101.

8 Wei G, Schaffner AE, Baker KM, et al. Anticancer Res 2003; 23:21732178.

9 Velasco G, Grkovic S, Ansieau S. J Biol Chem 2006; 281:1654616550 .

10 Holm L, Rosenstrom P. Nucleic Acids Res 2010; 38:W545-549.

11 Letunic I, Doerks T, Bork P. Nucleic Acids Res 2012; 40:D302-D305.

12 Brien GL, Gambero G, O'Connell DJ, et al. Nat Struct Mol Biol 2012; 19:1273-1281.

(Supplementary information is linked to the online version of the paper on the Cell Research website.) 